\title{
Adhesion and friction between individual carbon nanotubes measured using force-versus-distance curves in atomic force microscopy
}

\author{
Bharat Bhushan* and Xing Ling \\ Nanoprobe Laboratory for Bio- \& Nanotechnology and Biomimetics (NLB2), The Ohio State University, 201 West 19th Avenue, \\ Columbus, Ohio 43210-1142, USA
}

(Received 21 April 2008; revised manuscript received 2 June 2008; published 28 July 2008)

\begin{abstract}
The adhesion and friction between individual nanotubes was investigated in ambient using a dynamic atomic force microscope (AFM) operating in force-calibration mode to capture force-versus-distance curves. A multiwalled carbon nanotube (MWNT) tip attached to a conventional AFM probe was brought into contact with and then ramped in vertical direction against a single-walled carbon nanotube (SWNT) bridge suspended over a $2-\mu \mathrm{m}$-wide trench. The interaction between nanotubes altered the oscillation amplitude, phase lag, and average deflection of AFM cantilever, from which the interacting forces between nanotubes are quantitatively derived. During ramping, a stick-slip motion was found to dominate the sliding between the nanotubes. The stick was attributed to the presence of high-energy points, such as structural defects or coating of amorphous carbon, on the surface of the MWNT tip. The coefficients of static friction and shear strength between nanotubes were evaluated to be about 0.2 and $1.4 \mathrm{GPa}$, respectively. They are about 2 orders of magnitude larger than the kinetic counterparts. The kinetic values are on the same order as that measured previously by sliding a MWNT tip across a SWNT bridge in lateral direction.
\end{abstract}

DOI: 10.1103/PhysRevB.78.045429 PACS number(s): 46.55.+d, 68.35.Np, 61.48.De, 07.79.Lh

\section{INTRODUCTION}

With their remarkable mechanical and electrical properties, ${ }^{1,2}$ carbon nanotubes (CNTs) are used to blend with polymers, metals, and ceramics ${ }^{3}$ or weaved into fibers and sheets ${ }^{4-8}$ for fabricating macroscale materials with extraordinary strength and toughness and good electrical conductivity. The mechanical strength of these materials critically depends on the mechanical and tribological properties of CNTs, and the small interfacial shear strength between nanotubes and matrix was found to be a limiting factor for enhancing the composite/fiber strength due to inefficiency in interfacial stress transfer. ${ }^{3,7,9,10}$ To overcome this problem, efforts have been made to introduce defects on the nanotubes by electron irradiation ${ }^{11}$ or chemical bonds between nanotubes and matrix ${ }^{3,10}$ in attempts to increase the friction and cross-linking between nanotubes and matrix. However, without quantification of the forces between nanotubes on an individual nanotube level, it is difficult to precisely evaluate how the modification on the structures of individual nanotubes influences the performance of composite/fiber at a macroscale.

The direct nanotribological characterization between two nanotubes is scarce. Very recently our group had addressed this issue by scanning an atomic force microscope (AFM) tip with a multi-walled carbon nanotube (MWNT) welded to its end across a single-walled carbon nanotube (SWNT) bridge suspended on a microtrench in lateral direction. ${ }^{12}$ The MWNT tip had its outer shells partially stripped possibly with a sword-in-sheath breaking mechanism due to repetitive scans inside the trench, ${ }^{13}$ leaving a fresh, contamination-free, and defects-minimized surface. The coefficient of kinetic friction between the stripped MWNT tip and the SWNT bridge was estimated to be as low as 0.006 , consistent with the value measured on graphite. ${ }^{14,15}$

Herein, we reinvestigate the friction between individual nanotubes using a pristine MWNT tip freshly grown using catalytic chemical vapor deposition. ${ }^{16}$ Its surface should bear the original structure as formed in the chemical-vapor deposition (CVD) environment, allowing us to compare with the stripped MWNT tip to gain further insights into the dependence of friction on the surface structure of nanotubes. Also the methodology for measuring the friction between nanotubes is modified to accommodate the increased stiffness of the pristine MWNT tip by operating the AFM in forcecalibration mode to capture force-versus-distance curves. In this mode, the MWNT tip is ramped in a vertical direction against a SWNT bridge instead of scanning across it in a lateral direction. The latter is inappropriate for a stiff MWNT tip as it might damage the SWNT bridge during scanning. ${ }^{12}$ It is well known that on a flat surface, a technique referred to as friction loop measurement is employed as a standard method for measuring friction at the nanoscale using AFM. Here, during the ramping of a MWNT tip in the vertical direction against a SWNT bridge, in the ideal case that the MWNT tip is straight and nondeformable, only the friction would cause the vertical deflection of the AFM cantilever to change. The vertical deflection reverses its sign when the MWNT tip switches its ramping direction from extending to retracting (vice versa) and thus forms a friction loop, too, from which the friction between nanotubes is derived. In view of its similarities with the conventional friction loop measurement, we refer our method as vertical friction loop measurement.

In addition, during the ramping, the AFM cantilever is driven constantly by an embedded piezodriver at its resonance frequency. This enables us to acquire two additional signals, the oscillation amplitude and the phase lag of the AFM cantilever with respect to the driving force. Using a point-mass model to describe the cantilever dynamics, two terms causing the attenuation of amplitude, the interaction stiffness conservative in nature and the damping coefficient dissipative in nature, are extracted from the combination of 
MWNT tip attached to Si tip

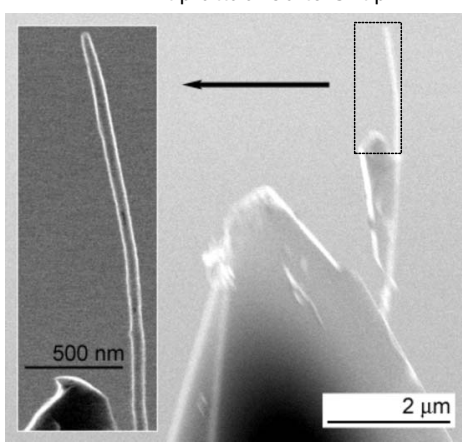

FIG. 1. The SEM image shows the morphology of the MWNT tip.

amplitude and phase data. By treating the SWNT bridge as an elastic spring with its ends pinned at each edge of the trench, the elastic restoring force and the stiffness of the SWNT bridge are functions of the displacement of the SWNT bridge under the force applied from the MWNT tip. They can be further related to the measured vertical force and the interaction stiffness through the angle of the displacement. With these relationships, the distance and the angle of displacement of the SWNT bridge during ramping are calculated, which enables precise evaluation of the intershell adhesive and friction forces between nanotubes by taking into account the effect of contact geometry. The friction force is compared to the adhesive force to produce the coefficient of friction between nanotubes. The contact size and the shear strength between nanotubes are also derived using a continuum model.

\section{EXPERIMENTAL}

The synthesis of SWNT bridges and fabrication of MWNT tip were described in detail previously. ${ }^{12}$ In short, SWNT bridges, suspended on top of polycrystalline silicon trenches of $2 \mu \mathrm{m}$ wide and $1.5 \mu \mathrm{m}$ deep, were synthesized by catalytic thermal CVD in a low-pressure furnace. ${ }^{17,18}$ The MWNT tip was prepared by mounting an individual MWNT, grown by CVD on a Pt wire coated with a liquid catalyst solution, to the tip of a conventional AFM probe using a micromanipulator operated under an inverted microscope. ${ }^{16}$ The scanning electron microscopy (SEM) image for the MWNT tip used here is displayed in Fig. 1. The normal spring constant $k_{z}$ of the MWNT probe was calibrated to be $1.26 \mathrm{~N} / \mathrm{m}$ using the Sader method. ${ }^{19}$

To measure the adhesion and friction between nanotubes, the MWNT tip was brought into contact with and then ramped in the vertical direction against a SWNT bridge suspended on a microtrench using an AFM. The schematic for the experimental setup is presented in Fig. 2. The longitudinal axis of the trench edge was aligned with the long axis of the AFM cantilever. During tapping mode imaging, by manipulating the center and size of scan area, the MWNT tip was positioned inside the trench and close to one of its ends. The AFM was then switched to force-calibration mode to capture force-versus-distance curves. In this mode, the AFM

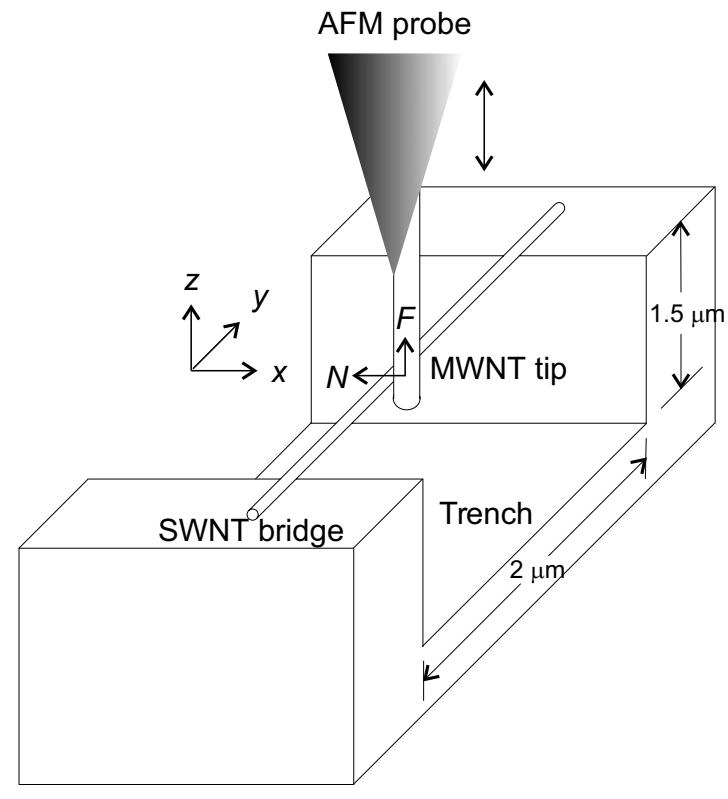

FIG. 2. Schematic drawing of the experimental setup for nanotribological measurements between individual nanotubes. The double arrow indicates the ramping direction ( $z$ direction). $F$ and $N$ indicate the friction and normal force between nanotubes.

probe is controlled to ramp in the vertical direction ( $z$ direction in Fig. 2), while its horizontal position relative to the trench is manipulated by offsetting in the $x$ and $y$ axes. To bring the MWNT tip into contact with the SWNT bridge, the $x$ offset was either gradually increased (the MWNT tip approaches the SWNT bridge from its right side) or decreased (approaching from the left side) in a step of $50 \mathrm{~nm}$, while the oscillation amplitude of AFM cantilever was closely monitored. After contact between the MWNT tip and the SWNT bridge was established, as indicated by an immediate drop of the oscillation amplitude, the horizontal position of the AFM probe was then fixed. By manipulating the two parameters, lowest ramping position and ramping size, the MWNT tip was controlled to ramp in the vertical direction at any height range above the trench bottom (within the $z$ limit of the scanner of $4.928 \mu \mathrm{m}$ ). The ramping rate was within $1-2 \mathrm{~Hz}$, and the ramping size varied from several tens of nanometers to several micrometers. The interaction between the MWNT tip and the SWNT bridge caused changes to the oscillation amplitude, phase lag, and average deflection of the AFM cantilever, from which the adhesion and friction between nanotubes are obtained using the strategies developed in Sec. III. The force sensitivity of the AFM cantilever is calibrated by performing force calibrations against the hard trench bottom. The temperature and relative humidity were $21 \pm 1{ }^{\circ} \mathrm{C}$ and $30 \pm 5 \%$, respectively.

\section{RESULTS AND DISCUSSION}

Force-versus-distance curves of the MWNT tip ramping against a SWNT bridge will be described first. The stick-slip motion dominates the sliding between this MWNT tip and the SWNT bridge, and the mechanism leading to this kind of motion is then discussed. Using the simultaneously measured 
MWNT tip below SWNT bridge
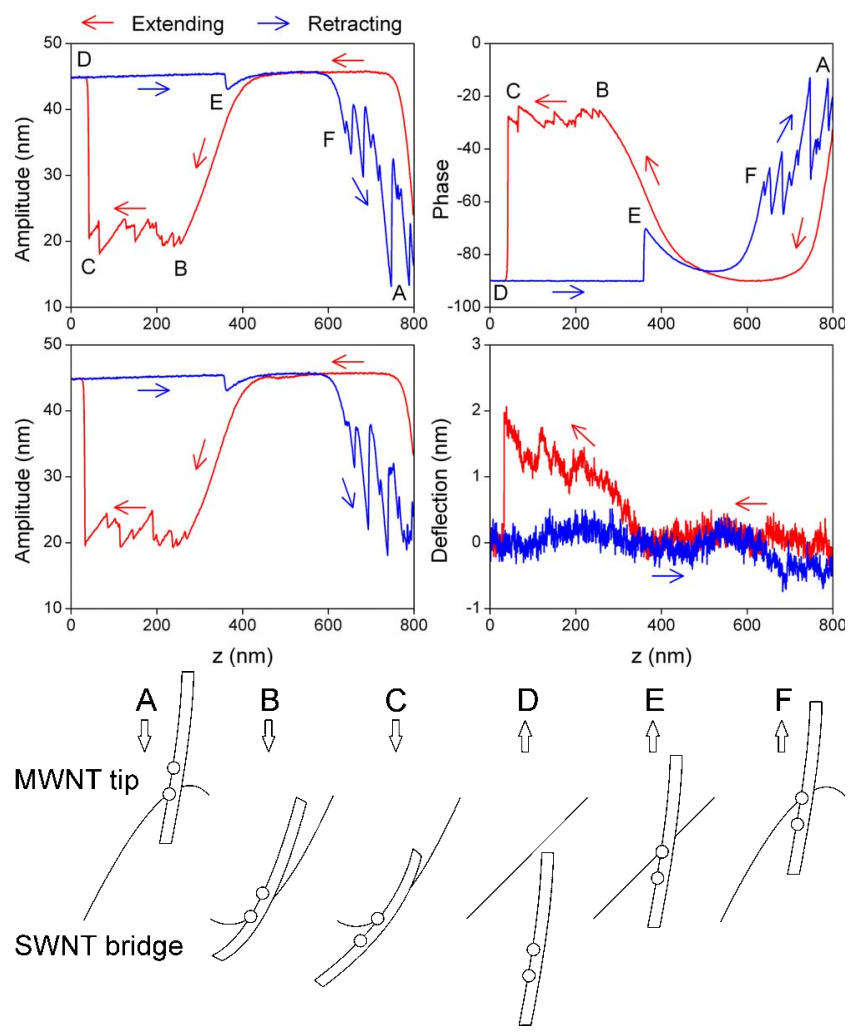

FIG. 3. (Color online) Typical force-versus-distance curves of the MWNT tip ramping in vertical direction against a SWNT bridge from below. Feature points are labeled in the top plots, and the corresponding contact geometries of nanotubes are illustrated in the bottom schematics. The arrows in the schematics indicate the ramping direction at those points.

oscillation amplitude and phase lag of the AFM cantilever, the conservative and dissipative components in the interaction between nanotubes are separated, and from the latter the average kinetic friction force between nanotubes is derived. A method for determining the contact geometry of nanotubes is introduced. The results are used for evaluating the adhesive and friction force between this MWNT tip and the SWNT bridge.

\section{A. Force-versus-distance curves of MWNT tip ramping against SWNT bridge and the stick-slip motion}

The force-versus-distance curves, in which the oscillation amplitude, phase lag and average deflection of AFM cantilever are plotted against its ramping distance, are shown in Fig. 3. They were captured on two consecutive scans due to insufficient data channels available. The MWNT tip was placed below the SWNT bridge and ramped at a height range of about -500 to $300 \mathrm{~nm}$ with respect to the trench top. Several feature points are marked in the curve, and for each point, the possible contact geometry between nanotubes is illustrated in the bottom schematics in Fig. 3. During continuous ramping cycles, at point A-the beginning of extending - the SWNT bridge was initially pulled up by the MWNT tip, as the oscillation amplitude of AFM cantilever was significantly lower than its free amplitude, and its deflection was negative (downward). The contact point between nanotubes at point $\mathrm{A}$ is denoted by the lower circle drawn onto the MWNT tip in the schematics. With progressive extending of the MWNT tip toward the trench bottom, the restoring force from the SWNT bridge was gradually reduced and then increased when the contact point moved below the trench top. The decrease and increase in the force between nanotubes led to the formation of an arc in the amplitude-distance curve between points A and B. After point $\mathrm{B}$, the restoring force exceeded the static friction between nanotubes, and the SWNT bridge began to slip up along the MWNT tip.

A stick-slip motion of the SWNT bridge was expected from the observed saw-tooth pattern in the amplitudedistance curve between points $\mathrm{B}$ and $\mathrm{C}$ in Fig. 3. At point C, the MWNT tip was extended to a threshold such that the adhesive force between nanotubes could no longer hold them together, and the two nanotubes detached from each other. The contact point between nanotubes at point of detachment $\mathrm{C}$ is denoted by a circle higher than the circle at which the nanotube initially contacted due to the slips between nanotubes. The response of the cantilever here gave a measure of the adhesive force. After detachment, the amplitude was immediately restored to the free amplitude and kept for the rest of the extending from point $\mathrm{C}$ to point $\mathrm{D}$. At point $\mathrm{D}$, the MWNT tip reversed its ramping direction and moved away from the trench bottom. Contact between the nanotubes appears to be reestablished at point $\mathrm{E}$, as immediate changes to both amplitude and phase are identified. The distance between point $\mathrm{C}$ and point $\mathrm{E}$, or the distance between the detachment and attachment of the nanotubes, clearly indicates to what extent the SWNT bridge was displaced. Although the same circle was used to denote the contact point in the schematic, it does not necessarily mean that the contact point at point $\mathrm{E}$ is exactly the same as that at point $\mathrm{C}$. The SWNT bridge was pulled with continuous retracting, and at point $\mathrm{F}$, where the contact point should be higher than the trench top, the static friction between nanotubes was overcome, and the SWNT bridge began to slip down along the MWNT tip. Again, a stick-slip motion dominated the sliding between the two nanotubes between points $\mathrm{F}$ and $\mathrm{A}$. At the end of the retracting, the contact point slipped to a point denoted by the lower circle in the schematic, where the next ramping cycle started.

Simply by increasing the ramping size by $200 \mathrm{~nm}$ while the lowest position of ramping is the same as before, the MWNT tip would break away from the SWNT bridge at the end of retracting and lay on top of it for the following ramping cycles. This allowed us to measure the force-versusdistance curves with the MWNT tip above the SWNT bridge, as shown in Fig. 4. The slight change in contact geometry dramatically altered the way in which the MWNT tip interacted with the SWNT bridge. This is evident by comparing Figs. 3 and 4. In a similar manner, feature points are marked on the curve, and corresponding contact geometries are illustrated. One can refer to the schematic in Fig. 4 for an intuitive picture of the ramping process. A few points worthy of mention are as follows. (1) A compressive force between the nanotubes was developed between points $\mathrm{C}$ and $\mathrm{D}$ in Fig. 4 

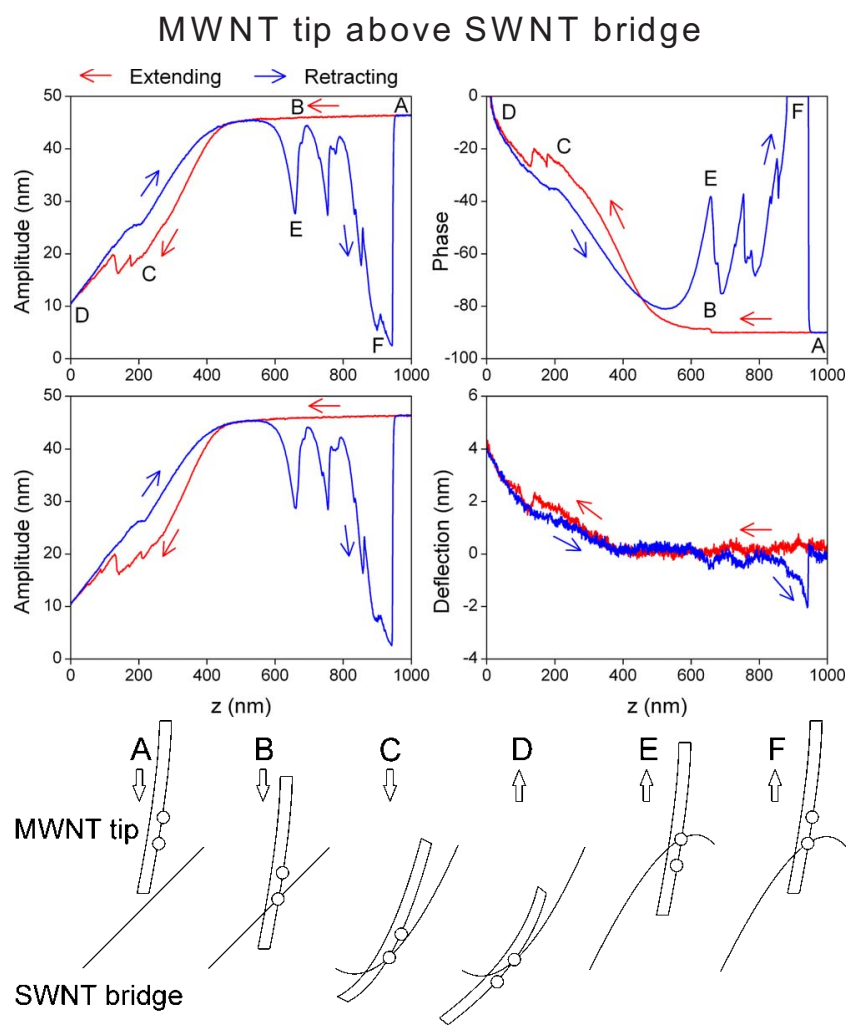

FIG. 4. (Color online) Typical force-versus-distance curves of the MWNT tip ramping in vertical direction against a SWNT bridge from above. Feature points are labeled in the top plots, and the corresponding contact geometries of nanotubes are illustrated in the bottom schematics. The arrows in the schematics indicate the ramping direction at those points.

instead of a pulling force in the region between point $\mathrm{B}$ and $\mathrm{C}$ in Fig. 3. Thus, the force worked collaboratively with the adhesive force between nanotubes to enhance the friction between them instead of weakening it in Fig. 3. (2) The detachment of nanotubes happened at the end of retracting in Fig. 4 instead of the end of extending in Fig. 3, where the contact points between nanotubes are expected to be different. (3) The saw-tooth pattern observed in Fig. 4 is significantly different from that in Fig. 3. In addition to different load between the nanotubes, the different sides which the MWNT tip used to contact the SWNT bridge are likely to be another contributor, indicating the stick-slip motion is surface sensitive.

The lack of atomic scale periodicity in the stick-slip motion (Figs. 3 and 4) suggests its origin from random highenergy points (HEPs) presented on the surface of nanotubes, most likely on the MWNT tip, rather than the periodic atomic lattice found previously. ${ }^{20}$ It is known that MWNTs grown in CVD environment are rich in structural defects and coating of amorphous carbon. ${ }^{1,2}$ The shape of the MWNT tip used here is far from the perfect cylinder in the SEM image of the MWNT tip shown in Fig. 1. During the ramping of the MWNT tip, the SWNT bridge tended to stick to a HEP unless the restoring force of the SWNT bridge grew strong enough to shear it away. After that the SWNT bridge slipped along the MWNT tip under the effect of the restoring force.

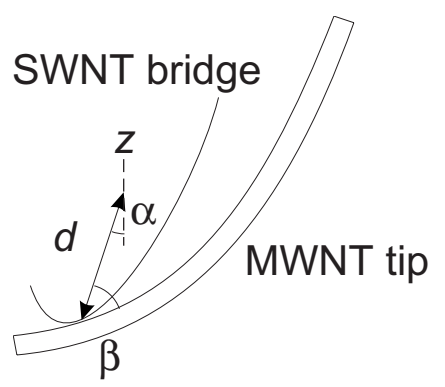

FIG. 5. Schematic illustration of the contact geometry of nanotubes. The SWNT bridge was displaced by a distance of $d$ and tilted from the vertical direction by an angle of $\alpha$. The angle between the displacement of the SWNT bridge and the MWNT tip is denoted as $\beta$.

In our case, the average distance between HEPs appeared to be smaller than the displacement of SWNT bridge, which led to resticking of the SWNT bridge to subsequent HEPs before the strain of SWNT bridge decreased to zero. The sticking force, i.e., static friction, between nanotubes was large enough to be detected as a variation of cantilever deflection (Figs. 3 and 4). In the ideal case that the MWNT tip is straight and nondeformable, only the friction force between nanotubes will contribute to the vertical deflection, as shown in Fig. 2. However, in reality, the MWNT tip here was tilted toward the $-x$ direction and could be deformed nonlinearly, and thus the vertical deflection always involved contributions from both normal and friction forces.

Figure 5 shows possible contact geometry with the MWNT tip below the SWNT bridge. The angles between the restoring force of the SWNT bridge $F$ (in the same direction as its displacement) and $z$ axis and the MWNT tip are $\alpha$ and $\beta$, respectively. The friction force $F_{F}$ is related to the vertical force $F_{V}$, which is an experimentally measurable quantity, by a conversion factor, $\cos \beta / \cos \alpha$. Generally speaking, to precisely calculate the friction force, one needs to determine these two angles. Later we will introduce a method for the evaluation of one of the angles, $\alpha$. At present we simply utilize the fact that when the contact point between nanotubes is much higher than the trench top, these two angles should be close to zero, leading to a conversion factor of about 1 . This means that we can use the cantilever deflection data from the right portion of force-versus-distance curves, where the contact point is higher than the trench top to calculate the friction force between nanotubes. Seventeen forceversus-distance curves captured with the MWNT tip below the SWNT bridge are summarized in Fig. 6(a) by plotting the vertical force (normal spring constant $\times$ verticle deflection) versus the normalized amplitude. The left and right portions of force-versus-distance curves split into the upper and lower branches in Fig. 6(a) with the left portion corresponding to the upper branch and vice versa. The static friction force estimated from the lower branch varied between 0 and 1.3 $\mathrm{nN}$. The large noise in the deflection data, including thermal noise on the order of $0.1 \mathrm{nN}$ (Ref. 12) and strong laser interference, prevents an accurate estimation of the lower limit of the static friction force, considering the slippage between nanotubes could happen at point $\mathrm{F}$ in Fig. 3 where the amplitude is close to the free amplitude. 


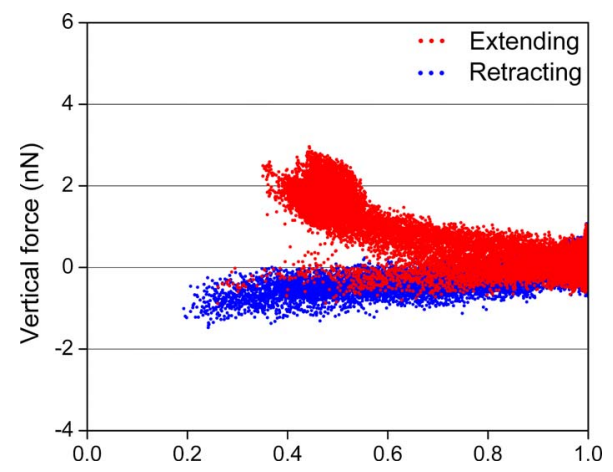

(a) MWNT tip below SWNT bridge

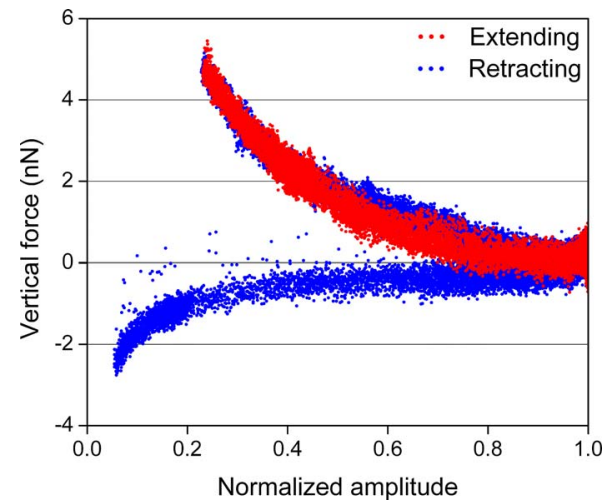

(b) MWNT tip above SWNT bridge

FIG. 6. (Color online) The interaction between nanotubes is partially detected as vertical deflection of AFM cantilever, from which the vertical force is calculated and plotted against normalized amplitude using (a) 17 force-versus-distance curves with the MWNT tip below the SWNT bridge and (b) 12 force-versusdistance curves with the MWNT tip above the SWNT bridge.

The detachment of the nanotubes near the end of extending in Fig. 3 and the end of retracting in Fig. 4 allows estimation of the adhesive force between nanotubes at different contact points. In Fig. 6(b), 12 force-versus-distance curves captured with the MWNT tip above the SWNT bridge are also summarized. The vertical forces are almost the same and close to $3.0 \mathrm{nN}$ for the highest point in Fig. 6(a) and the lowest point in Fig. 6(b). This is an unexpected result since the conversion factor for the adhesive force from the vertical force, $\sin \beta / \cos \alpha$, would be different when the SWNT bridge detached from the MWNT tip using different sides and at different ramping distances. This interesting coincidence will be addressed quantitatively when the method for determining the angle $\alpha$ is introduced.

\section{B. Interaction stiffness, the damping coefficient, and the kinetic friction force between nanotubes}

The dominant interaction between the MWNT tip used here and the SWNT bridge was stick-slip. While the MWNT tip stuck to the SWNT bridge, there was no sliding between nanotubes, and thus no kinetic friction could occur. Only during the occurrence of slip between nanotubes might the kinetic friction be detected from the amplitude attenuation using the method developed by Bhushan et al. ${ }^{12}$ The average distance between HEPs appears to be small, and the shifting motion of SWNT bridge between HEPs is extremely fast due to its small mass and relatively large force so that the slips in the middle of sticks are expected to be transient, as sharp kinks are observed in Figs. 3 and 4. Therefore, the effect of kinetic friction on the amplitude attenuation, if detectable, would be small. Then a question arises: what caused the large amplitude attenuation observed in Figs. 3 and 4? To answer this question, the contributions to amplitude attenuation from different sources will be quantitatively analyzed below using a treatment proposed by Jai et al. ${ }^{21}$ The interaction between the nanotubes is separated into two terms: one is conservative and proportional to the tip displacement $\left(-k_{\text {int }} z\right)$, and the other is dissipative and proportional to the tip velocity $\left(-\gamma_{\text {int }} \dot{z}\right)$, where $k_{\text {int }}$ and $\gamma_{\text {int }}$ are the interaction stiffness and damping coefficient, respectively. Using a point mass model to describe the steady-state motion of the AFM probe, which is driven under a frequency of $\omega$ with a resonance frequency of $\omega_{0}$, a quality factor of $Q_{0}$, and a free amplitude of $A_{0}$, the normalized interaction stiffness is expressed as ${ }^{21}$

$$
\frac{k_{\text {int }}}{k_{z}}=\left(\frac{\omega}{\omega_{0}}\right)^{2}-1+\frac{\cos \varphi+A_{0} /\left(A \sqrt{1+Q_{0}^{2}}\right)}{A \sqrt{1+Q_{0}^{2}} / A_{0}+2 \cos \varphi+A_{0} /\left(A \sqrt{1+Q_{0}^{2}}\right)},
$$

where $k_{z}$ is the spring constant of the cantilever, and $A$ and $\varphi$ are the attenuated oscillation amplitude and phase lag of AFM cantilever. The normalized damping coefficient is given by ${ }^{21}$

$$
\frac{\gamma_{\mathrm{int}}}{\gamma_{0}}=-\frac{\omega_{0}}{\omega} \frac{Q_{0} \sin \varphi}{A \sqrt{1+Q_{0}^{2}} / A_{0}+2 \cos \varphi+A_{0} /\left(A \sqrt{1+Q_{0}^{2}}\right)}-1,
$$

where $\gamma_{0}$ is the bulking damping coefficient in air.

Using Eqs. (1) and (2), the normalized interaction stiffness and the damping coefficient are calculated from various combinations of normalized oscillation amplitude $A / A_{0}$ and phase lag $\varphi$ and plotted as contour plots in Fig. 7. The measured amplitude and phase with the MWNT tip below and above the SWNT bridge are mapped into the contour plots for straightforward evaluations of the interaction stiffness and damping coefficient. It can be concluded from Fig. 7 that the interaction between nanotubes is dominated by the conservative term, the interaction stiffness. During the interaction of nanotubes, the SWNT bridge acted as an elastic spring, and its stiffness was added to the AFM cantilever through the MWNT tip. The dissipative term is small as all data basically follow the zero contour line of damping coefficient. The deviation of the bottom data from the zero contour line should be an artifact associated with the inaccurate measurements of amplitude and phase data at small amplitude. Moreover, the difference between the extending and retracting data is small, although load and contact geometries are expected to be significantly different, suggesting the deviation results from measurement errors. The domination of interaction stiffness in the interaction between nanotubes confirms the stick-slip motion as the sliding mechanism be- 


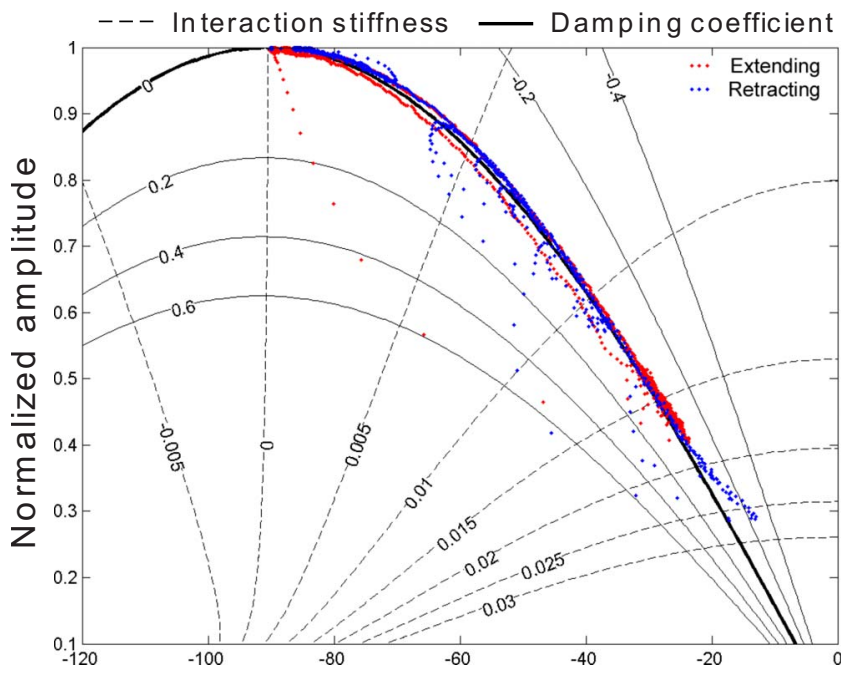

(a) MWNT tip below SWNT bridge

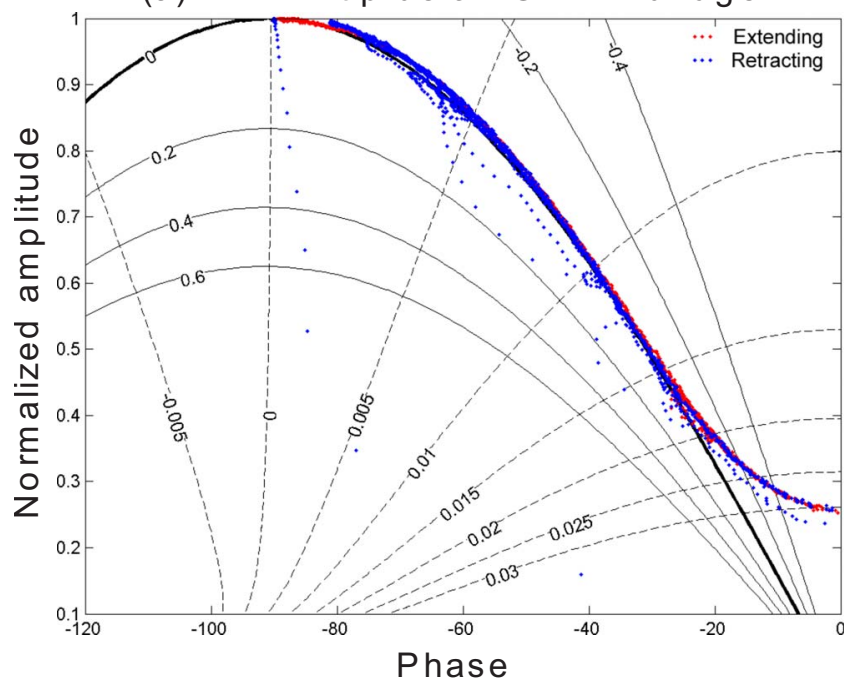

(b) MWNT tip above SWNT bridge

FIG. 7. (Color online) The relative magnitude of interaction stiffness and damping coefficient for the interaction between nanotubes are determined by mapping (a) the data in the top figures of Fig. 3 and (b) in the top figures of Fig. 4 into the contour plots of interaction stiffness and damping coefficient against normalized amplitude and phase. The solid lines represent the damping coefficient and the dashed lines represent the interaction stiffness. The data basically follow the zero contour line of damping coefficient highlighted by a thick solid line.

tween this MWNT tip and the SWNT bridge since only during sticking could the stiffness of SWNT bridge be effectively transferred to the AFM cantilever.

The average kinetic friction between nanotubes is estimated from the amplitude and phase data using a formula developed by Bhushan et al., ${ }^{12}$

$$
F_{F}=\frac{\pi k_{z}\left(A_{0} \sin \varphi-A\right)}{4 Q} .
$$

It can also be obtained by integrating the instantaneous power dissipation over one oscillation period from Eq. (2) with an approximation that $\gamma_{\text {int }} / \gamma_{0}=-A_{0} \sin \varphi / A-1$ under high $Q_{0}$. The results are plotted against normalized amplitude in Fig. 8. Except for a few transient points, the kinetic friction force never exceeded $20 \mathrm{pN}$. The kinetic friction force is on the same order as the value previously measured by Bhushan et al. ${ }^{12}$ by sliding a MWNT tip across a SWNT bridge in lateral direction, as expected for shearing an incommensurate contact between graphite sheets. ${ }^{22}$ Note that unphysical negative frictions are generated at small amplitude due to inaccurately measured amplitude and phase data. However, the difference between the forces during the extending and retracting is still within $20 \mathrm{pN}$ for the data at small amplitude.

\section{Determination of the contact geometry for calculating the adhesive force between nanotubes}

This section is devoted to the determination of the displacement of the SWNT bridge (Fig. 5), from which the conversion factor to obtain the adhesive force from the vertical force can be calculated. Due to the complexity involved in the three-dimensional contact between nanotubes, a few simplifications need to be made before the displacement of the SWNT bridge becomes computable with present data. First, the SWNT bridge is assumed to be perpendicular to the trench wall, and the MWNT tip is not tilted in the $y$ direction. Second, the MWNT tip is assumed to contact the SWNT bridge at the center of the bridge. These two assumptions are necessary to reduce the contact problem to two dimensions, i.e., the $x z$ plane. Third, the MWNT tip is assumed to be much stiffer than the SWNT bridge so that its contribution to the interaction stiffness can be neglected. With these assumptions, the unknown quantities, restoring force and stiffness of the SWNT bridge, are directly related to the measurable quantities, vertical force and interaction stiffness, through the displacement of the SWNT bridge. Referring to Fig. 5, the interaction stiffness is a component of the stiffness of the SWNT bridge, $k_{\text {int }}=k \cos ^{2} \alpha$, and the vertical force is a component of the restoring force, $F_{V}$ $=F \cos \alpha$. By treating the SWNT bridge as an elastic string, the restoring force is a simple function of the displacement of the SWNT bridge, derived from the equations given by Walters et al. ${ }^{23}$

$$
F=2 E A\left(\bar{d}-\frac{\bar{d}}{\sqrt{\bar{d}^{2}+1}}\right),
$$

where $A$ and $\bar{d}$ are the cross-sectional area and the normalized displacement of SWNT bridge $(\bar{d}=2 d / g$, where $g$ is the gap width of the trench). Its derivative gives the stiffness for the SWNT bridge,

$$
k=\frac{4 E A}{g}\left[1-\frac{1}{\left(1+\bar{d}^{2}\right)^{3 / 2}}\right] .
$$

The value of $F_{V} / k_{\text {int }}^{1 / 2}$ is a function of $\bar{d}$ only. For each data point in Figs. 3 and $4, F_{V}$ is estimated directly from the measured vertical deflection, and $k_{\text {int }}$ is estimated from the amplitude by assuming $\gamma_{\text {int }}=0$ using Eqs. (1) and (2). The displacement is then calculated numerically from the value 


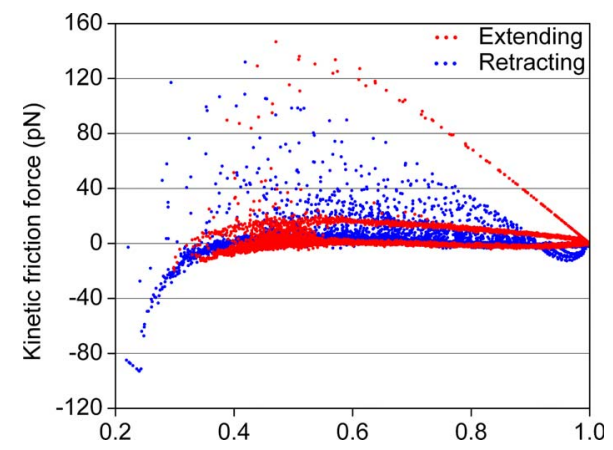

(a) MWNT tip below SWNT bridge

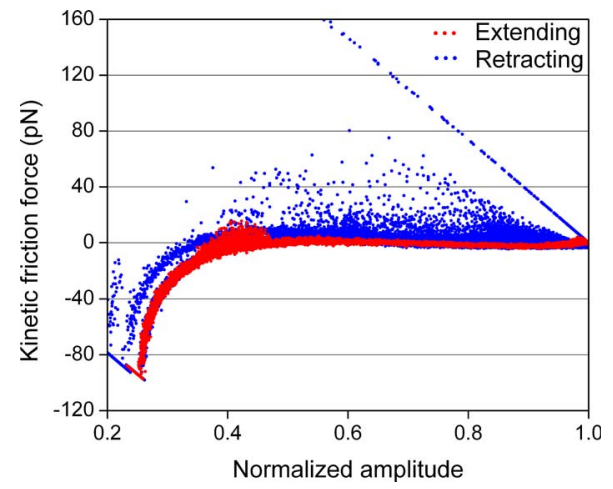

(b) MWNT tip above SWNT bridge

FIG. 8. (Color online) Plots of kinetic friction force between nanotubes against normalized amplitude as calculated from the combination of amplitude and phase data using (a) nine forceversus-distance curves with the MWNT tip below the SWNT bridge and (b) 14 force-versus-distance curves with the MWNT tip above the SWNT bridge.

of $F_{V} / k_{\text {int }}^{1 / 2}$ from Eqs. (4) and (5). By substituting the displacement into Eq. (4), the restoring forces are calculated, which are then compared to the vertical force to obtain the angle $\alpha$.

The results are shown in Fig. 9. It is clear that in Fig. 9(a) the angle is close to zero $(\cos \alpha \approx 1)$ for the right portion of force-versus-distance curves shown in Fig. 3, justifying its previous use for evaluating the friction force between nanotubes. Unrealistic values of $\cos \alpha$ greater than 1 are produced in Fig. 9(b), which also result from the inaccurate measurements of amplitude and phase data at small values of amplitude. The displacement of the SWNT bridge is about $150 \mathrm{~nm}$ for the highest point in Fig. 9(a), roughly matching the distance between points $\mathrm{C}$ and $\mathrm{E}$ in Fig. 3. Their difference is attributed to the deformation of the MWNT tip at point $\mathrm{C}$.

\section{Determination of the adhesive force, friction coefficient, and shear strength between nanotubes}

The adhesive force between nanotubes is related to the vertical force by a conversion factor of $\sin \beta / \cos \alpha$. With the MWNT tip below the SWNT bridge, the detachment of nanotubes occurred near the end of extending at point $\mathrm{C}$ in Fig. 3. At point $\mathrm{C}$, the MWNT tip was pulled up by the SWNT bridge, and the angle between the nanotubes, $\beta$, would increase with this geometry. Also the adhesive force between nanotubes is counterbalanced by the pulling force


(a) MWNT tip below SWNT bridge
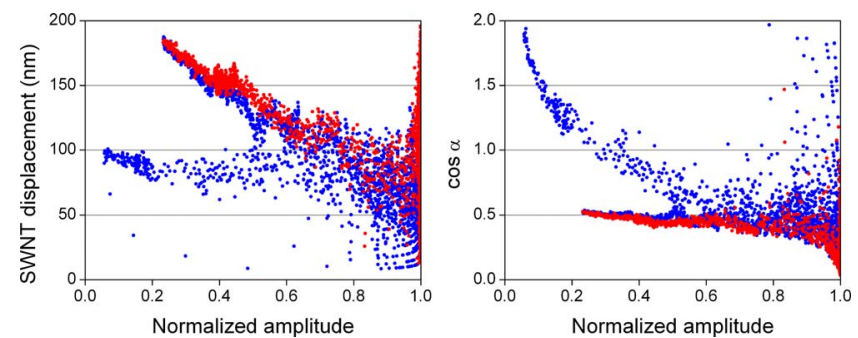

(b) MWNT tip above SWNT bridge

FIG. 9. (Color online) The contact geometry between nanotubes is partially determined and the distance (left column) and angle (right column) of the displacement of SWNT bridge are plotted against normalized amplitude. The plots are calculated from (a) Fig. 3 and (b) Fig. 4. The results are used for precise evaluation of the adhesive and friction force between nanotubes.

from the SWNT bridge (which is the reason the nanotubes detached from each other), leading to an almost zero friction force at point $\mathrm{C}$. However, the vertical force is large at this point, close to $3.0 \mathrm{nN}$, which means that the angle $\beta$ must be close to $90^{\circ}$ to minimize its shear component, which is balanced by the nearly zero friction force. In addition, we estimate $\cos \alpha$ of 0.5 for data at point $\mathrm{C}$, which leads to a conversion factor of 2 , and thus the adhesive force between nanotubes should be $6.0 \mathrm{nN}$ from the measured vertical force of $3.0 \mathrm{nN}$. In Fig. 9(b), the MWNT tip detached the SWNT bridge from above, and the angles $\alpha$ and $\beta$ would be both close to zero. Thus the adhesive force between nanotubes should be smaller than the measured vertical force of $3.0 \mathrm{nN}$ for a conversion factor smaller than 1 . The difference between the adhesive forces indicates that the MWNT tip might detach from the SWNT bridge using its tip end when it was placed above the SWNT bridge instead of using its shell when it was placed below the SWNT bridge. The adhesive force between the end of the MWNT tip and the shell of SWNT bridge was reported to be $0.7 \mathrm{nN}$ by Bhushan et al., ${ }^{12}$ about an order of magnitude smaller than the adhesive force between the shells of the nanotubes measured here, possibly due to the small radius of curvature at the MWNT tip end.

By comparing the friction force to the adhesive force, an upper limit for the coefficient of static friction is estimated to be 0.2 , about 2 orders of magnitude larger than the coefficient of kinetic friction of 0.003 . The latter is on the same order as the value reported previously for the stripped MWNT tip. ${ }^{12}$ The HEPs, which led to the $1 \mathrm{nN}$ static friction during the sliding between nanotubes, were reported to increase the interlayer sliding friction inside MWNT to the 1 $\mathrm{nN}$ range as well. ${ }^{24}$ While the HEPs in the interlayer sliding experiments could be dynamic due to e-beam radiation, ${ }^{24}$ the 
HEPs on this MWNT tip appear to be intrinsic and permanent as the repeatability of force-versus-distance curves were reasonably good. This finding highlights the importance of the surface structure of nanotubes on their nanotribological properties.

The interface energy and shear strength between nanotubes are estimated using the Johnson-Kendall-Roberts model. ${ }^{25}$ The MWNT has a diameter of $70 \mathrm{~nm}$, and the SWNT has an average diameter of $1.43 \mathrm{~nm} .{ }^{18}$ The measured adhesion force of $6.0 \mathrm{nN}$ corresponds to a work of adhesion between nanotubes of $0.25 \mathrm{~N} / \mathrm{m}$, as estimated using the equation

$$
W_{A}=\frac{2 F_{A}}{3 \pi \sqrt{r_{1} r_{2}}},
$$

where $r_{1}$ and $r_{2}$ are the radii of the MWNT tip and the SWNT bridge, respectively. This value agrees with the cohesive energy of graphite measured using other methods, ${ }^{26,27}$ justifying the use of the contact geometry estimation method. The contact area between nanotubes at zero loads is given by

$$
A=\pi\left(6 \pi W_{A} r_{1} r_{2} / K\right)^{2 / 3},
$$

where $K$ is the composite Young's modulus, given as $K$ $=2 E / 3\left(1-v^{2}\right)$, where $v$ is the Poisson ratio of nanotube, assumed to be 0.3 . Using a reported value of $0.22 \mathrm{~N} / \mathrm{m}$ as the work of adhesion between nanotubes, ${ }^{27}$ a contact area at zero force of $0.85 \mathrm{~nm}^{2}$ is obtained. The shear strength obtained by dividing the static friction with the contact area could be as high as $1.4 \mathrm{GPa}$, which is about 2 orders of magnitude larger than the shear strength calculated using kinetic friction. $^{12}$

\section{CONCLUSION}

In this paper, a method, referred to here as vertical friction loop measurement, was proposed to characterize the adhesion and friction properties between individual carbon nanotubes using the force-calibration-mode AFM in ambient. A MWNT tip attached to a conventional AFM probe was ramped in the vertical direction against a SWNT suspended on a microtrench at crossed geometry. The adhesion and friction between nanotubes were evaluated from the response of AFM cantilever during ramping.

During ramping, a stick-slip motion was found to dominate the sliding between the nanotubes. We attribute the stick to the presence of HEPs on the surface of this MWNT tip, associated with structural defects or coating of amorphous carbon. The stick was broken once the restoring force of the SWNT bridge became large enough to overcome the static friction between nanotubes, and the nanotubes began to slip against each other. The slip events were transient due to the fast shifting of the SWNT bridge between the HEPs separated by small distance.

The dynamics of the AFM cantilever were analyzed using a point-mass model, and the amplitude attenuation of the AFM cantilever during the interaction of nanotubes was found to be primarily attributed to the increase in the effective cantilever stiffness. The dissipative contribution from the kinetic friction between nanotubes to the amplitude attenuation is significantly smaller than the contribution from the conservative interaction stiffness.

The SWNT bridge acts as an elastic spring, and its restoring force and stiffness are functions of its displacement and related to the measured vertical force and interaction stiffness. By analyzing their relations, the distance and angle of displacement were calculated. They are used to calculate the adhesive and friction forces between nanotubes by taking into account the contact geometry between nanotubes.

The intershell adhesive force was found to be $6.0 \mathrm{nN}$, from which the work of adhesion between nanotubes is estimated to be $0.25 \mathrm{~N} / \mathrm{m}$, consistent with the value measured using different methods. The static friction was estimated to be as high as $1.3 \mathrm{nN}$ from the vertical deflection of cantilever during the stick-slip motion. Dividing the static friction by the adhesive force yields a coefficient of static friction of 0.2 , and the shear strength between nanotubes evaluated from the static friction is $1.4 \mathrm{GPa}$. The average kinetic friction between nanotubes was estimated to be smaller than $20 \mathrm{pN}$, which leads to a kinetic friction coefficient of 0.003 , comparable to the value reported before. The coefficient of static friction is about 2 orders of magnitude larger than that of the coefficient of kinetic friction, emphasizing the importance of the surface structure of nanotubes on their nanotribological properties.

\section{ACKNOWLEDGMENTS}

The authors gratefully acknowledge Alain Jungen for providing the SWNT sample, Cattien V. Nguyen for providing the MWNT probe, and Kwang Joo Kwak for SEM measurements.

\footnotetext{
*Author to whom correspondence should be addressed; bhushan.2@osu.edu

${ }^{1}$ M. S. Dresselhaus, G. Dresselhaus, and P. Avouris, Carbon Nanotubes: Synthesis, Structure, Properties, and Applications (Springer, New York, 2000).

${ }^{2}$ B. Bhushan, Springer Handbook of Nanotechnology (Springer, Berlin, 2007).

${ }^{3}$ E. T. Thostenson, Z. F. Ren, and T. W. Chou, Compos. Sci. Technol. 61, 1899 (2001).
}

${ }^{4}$ B. Vigolo, A. Penicaud, C. Coulon, C. Sauder, R. Pailler, C. Journet, P. Bernier, and P. Poulin, Science 290, 1331 (2000).

${ }^{5}$ K. L. Jiang, Q. Q. Li, and S. S. Fan, Nature (London) 419, 801 (2002).

${ }^{6}$ A. B. Dalton, S. Collins, E. Munoz, J. M. Razal, V. H. Ebron, J. P. Ferraris, J. N. Coleman, B. G. Kim, and R. H. Baughman, Nature (London) 423, 703 (2003).

${ }^{7}$ M. Zhang, K. R. Atkinson, and R. H. Baughman, Science 306, 1358 (2004). 
${ }^{8}$ M. Zhang, S. L. Fang, A. A. Zakhidov, S. B. Lee, A. E. Aliev, C. D. Williams, K. R. Atkinson, and R. H. Baughman, Science 309, 1215 (2005).

${ }^{9}$ R. H. Baughman, A. A. Zakhidov, and W. A. de Heer, Science 297, 787 (2002).

${ }^{10}$ J. N. Coleman, U. Khan, and Y. K. Gun'ko, Adv. Mater. (Weinheim, Ger.) 18, 689 (2006).

${ }^{11}$ A. Kis, G. Csanyi, J. P. Salvetat, T. N. Lee, E. Couteau, A. J. Kulik, W. Benoit, J. Brugger, and L. Forro, Nat. Mater. 3, 153 (2004).

${ }^{12}$ B. Bhushan, X. Ling, A. Jungen, and C. Hierold, Phys. Rev. B 77, 165428 (2008).

${ }^{13}$ M. F. Yu, O. Lourie, M. J. Dyer, K. Moloni, T. F. Kelly, and R. S. Ruoff, Science 287, 637 (2000).

${ }^{14}$ J. A. Ruan and B. Bhushan, J. Appl. Phys. 76, 5022 (1994).

${ }^{15}$ B. Bhushan, J. N. Israelachvili, and U. Landman, Nature (London) 374, 607 (1995).

${ }^{16}$ C. V. Nguyen, Q. Ye, and M. Meyyappan, Meas. Sci. Technol. 16, 2138 (2005).

${ }^{17}$ A. Jungen, C. Stampfer, J. Hoetzel, V. M. Bright, and C. Hierold, Sens. Actuators, A 130-131, 588 (2006).
${ }^{18}$ A. Jungen, S. Hofmann, J. C. Meyer, C. Stampfer, S. Roth, J. Robertson, and C. Hierold, J. Micromech. Microeng. 17, 603 (2007).

${ }^{19}$ J. E. Sader, J. W. M. Chon, and P. Mulvaney, Rev. Sci. Instrum. 70, 3967 (1999).

${ }^{20}$ C. M. Mate, G. M. McClelland, R. Erlandsson, and S. Chiang, Phys. Rev. Lett. 59, 1942 (1987).

${ }^{21}$ C. Jai, T. Cohen-Bouhacina, and A. Maali, Appl. Phys. Lett. 90, 113512 (2007).

${ }^{22}$ M. Hirano and K. Shinjo, Phys. Rev. B 41, 11837 (1990).

${ }^{23}$ D. A. Walters, L. M. Ericson, M. J. Casavant, J. Liu, D. T. Colbert, K. A. Smith, and R. E. Smalley, Appl. Phys. Lett. 74, 3803 (1999).

${ }^{24}$ A. Kis, K. Jensen, S. Aloni, W. Mickelson, and A. Zettl, Phys. Rev. Lett. 97, 025501 (2006).

${ }^{25}$ K. L. Johnson, K. Kendall, and A. D. Roberts, Proc. R. Soc. London, Ser. A 324, 301 (1971).

${ }^{26}$ R. J. Good, L. A. Girifalco, and G. Kraus, J. Phys. Chem. 62, 1418 (1958).

${ }^{27}$ L. X. Benedict, N. G. Chopra, M. L. Cohen, A. Zettl, S. G. Louie, and V. H. Crespi, Chem. Phys. Lett. 286, 490 (1998). 